\title{
Mechanism of Drug Resistance in Mycobacterium Tuberculosis
}

\author{
Ayanwale 0 Abraham*¹, Adabara U Nasiru ${ }^{1}$, Adeniyi K Abdulazeez ${ }^{2}$, Oyewole 0 Seun ${ }^{3}$ and David \\ W Ogonna ${ }^{4}$ \\ ${ }^{1}$ Department of Microbiology, Federal University of Technology, Nigeria
}

${ }^{2}$ Department of Animal Biology, Federal University of Technology, Nigeria

${ }^{3}$ Department of Microbiology, Obafemi Awolowo University, Nigeria

${ }^{4}$ Department of Biochemistry and molecular biology, Obafemi Awolowo University, Nigeria

*Corresponding author: Ayanwale O Abraham, Department of Microbiology, Federal University of Technology, Nigeria.

To Cite This Article: Ayanwale O Abraham, Mechanism of Drug Resistance in Mycobacterium Tuberculosis. 2020 - 7(5). AJBSR.MS.ID.001181. DOI: 10.34297/AJBSR.2020.07.001181.

Received: 眥 February 05, 2020; Published: 眥 February 26, 2020

\begin{abstract}
Summary
Mycobacterium tuberculosis the causative agent of tuberculosis has many intrinsic features which enable it to evade the activity of antibiotics. Many studies have been carried out to understand the mechanisms of drug resistance by this organism. An attempt was made in this write up to elucidate the various mechanism of drug resistance in M. tuberculosis, including its innate impermeable cell wall and mutation of specific genes. Drug resistance in Mycobacterium tuberculosis is not a product of a single homogeneous genetic unit. Rather it is as a result of frequent mutation in various genes which encode for resistance to antibiotics. Also, the slow metabolism during a prolonged dormant stage greatly enhances it resistance to drug, the waxy impermeable cell wall with the presence of numerous efflux pump are essential for withstanding the potency of antibiotics. Having an adequate knowledge on the molecular mechanisms of drug resistance in M. tuberculosis may be helpful in exploring new targets for drug development.
\end{abstract}

Keywords: Tuberculosis, Drug resistance, Antibiotic, Mycobacterium, Mechanism

\section{Introduction}

Tuberculosis (TB) is an infectious disease caused by Mycobacterium tuberculosis. Streptomycin, with high bactericidal activity was the earliest curative agent used in its treatment. However, drug-resistant strains began to emerge few years later. Initially, this was thought to result mainly from using only single drug, streptomycin, to treat the infection, prompting the use of multi-drug therapy for controlling the infection, but in recent decades multi-drug resistant (MDR) has emerged [1]. Furthermore, the reports gave by World Health Organization in 2010 reveal two other more classes which are, extensively- drug resistant (XDR), which is resistant to at least 4 of the core anti-TB drugs and totallydrug resistant (TDR) strains of TB. A type of TB resistance in people who are originally infected by the antibiotics resistant strain but have not used any anti-TB chemotherapy is regarded as the primary resistance, while a situation in which resistance developed due to inadequacy of treatment is referred to as acquired resistance $[2,3]$.

The complexity of the mechanisms used by M. tuberculosis in drug resistance has led scientists to studying M. tuberculosis at the molecular level. Various researchers have been able to identify some molecular features which have been attributed to drug resistance in the organism. When considering the mechanisms of drug resistance in $\mathrm{M}$. tuberculosis, it is imperative to understand the interplay between the molecular mechanisms, adaptive features and the innate attribute which play crucial role in resistant-MTB strain. Both [4] and Blair (2015), accentuated that drug resistance in Mycobacterium tuberculosis is not a single homogeneous biological unit, thus suggesting that the complexity and severity of resistance in the pathogen is due to some other intrinsic factors. 
This study aims to present findings on the mechanism of drug resistance in M. tuberculosis, presenting a comparative review of the molecular mechanism, the adaptive features and the innate mechanisms through which the organism develop resistance to antitubacular drugs.

\section{Mechanistic Innate Features of Drug Resistance Impermeable Cell Wall}

The fundamental characteristics of passive resistance to antibiotics in $\mathrm{M}$. tuberculosis are due to its impermeable cell wall [5]. M. tuberculosis has a cell wall that is composed of three main components, these are: mycolic acids, Wax-D and the cord factors [6]. The hydrophilic arabinogalactan layer ensures the impermeability of the cell wall to hydrophobic chemicals. This layer is further contained in hydrophobic mycolic acids which extremely impede the entrance of hydrophilic molecules [7]. This impermeability result in accumulation of antibiotics slowly around the cell, the accumulated drugs around the cell wall are slowly detoxify by different cellular component or by release of enzyme. [8], showed that $\beta$-lactams, which are inhibitive to the incorporation of peptidoglycan (rigidity structure) into the cell wall, are degraded by $M$. tuberculosis because it possesses $\beta$-lactamases, an enzyme that effectively degrade $\beta$-lactam antibiotics.

Danilchanka 0 et al. [7], reported the presence of an outer membrane channel protein CpnT in both $\mathrm{M}$. tuberculosis and M. bovis, which performs a dual role of nutrient uptake and selective susceptibility to antibacterial agents. The report showed that the CpnT mutant of M. bovis is more resistant to most antitubercular drugs, including the bactericidal nitric oxide, which is utilized in controlling M. tuberculosis infection in mice.

\section{Slow Metabolism Mechanism}

Bacteria with slow metabolic processes and long generation time are difficult target for most antibiotics i.e., bacteria that are metabolically active and replicate quickly are good target for antibiotics [9]. However, in M. tuberculosis, it is not still clear if the long generation time corroborate its drug resistance. According to [10], the long generation time in M. tuberculosis does not impact drug resistance ability on the organism. [11]. Also reported a negative association between drug resistance and generation time in the organism. However, the slow growth rate of M. tuberculosis has been reported to play a key role in its drug resistance, for example, unstable antibiotics such as carbapenems loses their activity at a faster rate than the mycobacterial growth rate [12], identified some specific genes which allow M. tuberculosis to grow in oxygen-deprived (stress) conditions, most of which are directly involved in triacylglycerol production. Triacylglycerol causes slowdown in the metabolic processes of Mycobacterium tuberculosis. This is mainly because triacylglycerol synthesis uses up acetyl CoA, which is an essential component in tricarboxylic acid cycle, a fundamental metabolic pathway [12]. This triacylglycerol is extensively produced by Mycobacterium tuberculosis when responding to diverse stress conditions such as oxygen deprivation, acidic $\mathrm{pH}$, and iron deficiency $[13,14]$, observed that the ability of the organism to grow under acidic condition is aided genetically and not only limited to physiological adaptations.

Also, evaluating the metabolic status of the organism at each stage of its pathogenesis is challenging since the organism can adapt to various microenvironment within the host. Furthermore, the extent of heterogeneity of infecting population in various cellular compartment within the same host adds to the challenges of targeting the metabolism processes [15] for therapeutic purpose.

\section{Possession of Numerous Efflux Pumps}

This protein channels play crucial role in the normal metabolism and physiology of the organism such as signaling molecules across the cell wall, and toxins, waste, and nutrient transportation [16]. Efflux pumps have also been shown to be adapted to drug resistance in M. tuberculosis. Multidrug efflux pumps pass through the inner and outer membranes and serve as an outlet for antibiotics from the cell [17]. Drug efflux pumps in M. tuberculosis have been found to possess regulatory protein systems; which controls efflux pump expression and thus specializing them for drug resistance roles [16].

\section{Molecular Mechanisms}

Acquisition of antibiotics resistance in M. tuberculosis has been shown to result from spontaneous mutation in several chromosomal genes, this frequent mutation has been found to confer alteration to the required interaction between each anti-tuberculosis drug and their specified target.

\section{Rifampicin}

This is a fundamental lipophilic annamycin chemotherapeutic agent initiated into the multi-drug treatment scheme of tuberculosis in the 1970s. It is usually combined with isoniazid as the first line chemotherapy in the treatment of tuberculosis [18]. Rifampicin is known to have an inhibitory effect against slow and actively growing tuberculosis [19]. Rifampicin mode of action is by binding to the $\beta$-subunit of the RNA polymerase, inhibiting the elongation of messenger RNA [20]. Rifampicin resistant strain of Mtb, usually serve as an indicator for multi-drug resistant tuberculosis, because these strains are not sensitive to all other tubercular drugs Comas et al., (2011) [21].

Resistance to rifampicin in M. tuberculosis have been found to be due to mutation in rpoB of RNA polymerase retarding affinity for rifampicin [20]. Some studies have been able to identify specific codon which can causes rifampicin resistance when mutation occur in them [22,23]. Report by [24] observed that most of the rifampicin resistant isolates, have a missense mutation and substitution 
of nucleotide at codon 526 and 531 of rpoB. [25], was also able to identify resistant strains with a form of mutation at 69-bp region and point mutation in rpoB alleles. [26] in his review work concluded that rifampicin resistance in tuberculosis is associated with nucleotide substitution at point 516,526 , or 531 of rpoB locus. The potency for rifampicin cross-resistance with rifamycin has been reported by several researchers, this has been characterized with conformational changes in codons (518 or 529) [27-29].

\section{Pyrazinamide}

Pyrazinamide is a first-line drugs, potent against nonreplicating persistent MTB [30]. PZA is also perform crucial function in reducing the relapse rate in tuberculosis; shortened the course of treatment from one year to six months [31] effective where there is resistance to rifampicin and isoniazid [32]. The proposed mechanism of action of pyrazinamide involves conversion of pyrazinamide to pyrazinoic acid, by the enzyme pyrazinamidase/ nicotinamidase coded by the pncA gene. The pyrazinoic acid disrupts the bacterial membrane energetics; membrane transport; formation of CoA, and acidification of the cytoplasm [32]. Most resistance to pyrazinamide has been associated with mutation in the pncA gene $[30,33]$. The mutation in pncA accounts for most of the resistance cases reported in MTB. Other targets and mechanism include: efflux pump [34] ribosomal protein S1 (RpsA) involved in trans-translation [31], Yang et al. (2015), identified mutation at the C-terminus of RpsA which is responsible for retarding the binding of POA to RpsA, thus making PZA inactive. 0

\section{Isoniazid}

Isoniazid and rifampicin form the core antibiotics in the treatment of tuberculosis. Isoniazid is usually present in the inactive form but their metabolism in the body convert them into a pharmacologically active form [35], Rouse et al. (2005). The metabolism of isoniazid to the active form occur via the activities of catalase/peroxidase enzyme KatG, coded by the KatG gene [36]. Once activated, it has been found to be a strong bacteristatic agent on metabolically and physiologically active M. tuberculosis, with a minimum inhibitory range of $(0.02 \mu \mathrm{g} / \mathrm{ml}$ to $0.06 \mu \mathrm{g} / \mathrm{ml})[37,38]$.

The mode of action of isoniazid resistance is complex and remains unclear, however, most isoniazid Mtb-resistant strains have been associated with mutation in KatG and inhA [39,40]. Mutations of the S315T of KatG is more common in isonazid resistant strains, mutation at this point causes the formation of isoniazid product with little affinity for isoniazid aduct [36]. Also, the conformational changes in inhA active site retard efficient binding to drug, drugs such as Ethionamide which have inhA as their target site are also affected (Ho et al., 2009). [40] who studied 11,411 Mtb isolates from 49 countries reported that $64 \%$ of all isoniazid resistant strain studied has mutation in katG315 while 19\% having mutation in the inhA-15 region. [41] reported that $88 \%$ of Mtb strains from patients with multidrug-resistant (MDR), and extensive drug resistant (XTR) tuberculosis in the Republic of Moldova had a katG 315T mutation. However, [38] was able to identify isoniazid resistant strains which do not harbor conformational changes in the KatG nor inhA.

\section{Ethambutol}

Part of the four-drug regimen anti-tuberculosis drug, it is combined with PZA, rifampicin, and isoniazid to prevent rifampicin resistance especially in cases where their isoniazid resistance is not detectable [42]. The mode of action of ethambutol involved interfering with synthesis of cell wall in the Mycobacterium tuberculosis [43]. However, several other formulated hypotheses on the mechanism of action of ethambutol include inhibition of the synthesis of spermidine [44], blockage of mycolic acid transfer to the cell wall [45], interfering with RNA metabolism [46], inhibition of the synthesis of phospholipid [45]. There is no clarity on the mechanism to ethambutol resistance, with the resistance mechanism initially related to mutation of the codon 306 in embB, $[47,48]$, however, certain studies have found M. tuberculosis with mutation at this same point to be susceptible to ethambutol $[49,50]$. Isoniazid resistant-MTB with mutation at the katG Ser315 were also found to concurrently exhibit resistance to ethambutol as well [48]. Polymorphism in embA, embC, and Mutations in embB497 and embB406, mutation in the codon 306 in embB have all been implicated in ethambutol resistance [48]. In 2013, Safi et al. proposed that mutation in ubiA (Rv3806c) resulted in high level of ethambutol resistance. [51], found mutations in ubiA in all the Ethambutol resistant Mtb he worked on, while [52], observed that ubiA mutations in Mycobacterium tuberculosis varies from one geographical location to another.

\section{Fluoroquinolones}

Fluoroquinone majorly the ciprofloxacins are chemotherapeutic agents used in the treatment of tuberculosis Rustomjee et al. (2008) [53], they have been found to demonstrate high antibacterial activity on Mycobacterium complexes and are majorly employed as combined therapy, it is inhibitory to DNA gyrase and topoisomerase IV [54]. Resistant to fluoroquinolone occur via mutation in the gyrA or gyrB [55]. Efflux pumps, pentapeptide proteins (MfpA) mediated regulation of gyrase, and the bacterial cell wall have been also implicated in fluoroquinone resistance in Mtb [34].

\section{Ethionamide}

These are antitubacular drugs that are structurally like isonaizid, in their normal state, they are inactive chemotherapeutic agents, and however, their metabolisms make them to be pharmacologically active, this activation requires a monooxygenase coded by ethA gene [56]. The mode of action of ethionamide by their inhibitory effect on the synthesis of mycolic acid in M. tuberculosis [57], the mechanism of resistance to ethionamide is largely unclear, however several report has associated the conformational changes in orf1 gene of inhA locus with ethionamide resistant strains [58], 
although certain resistant strains have also been found to harbor mutation at etaA / ethA, ethR [59].

\section{Kanamycin, Capreomycin, Amikacin, Viomycin}

These are second line antitubacular drugs which inhibit protein synthesis. Viomycin and Capreomycin are cyclic polypeptides, while, amikacin and kanamycin are aminoglycoside antibiotics [60]. Capreomycins and Viomycin are bacteriostatic antibacterial agents with a known mechanism of action of inhibiting translation reactions through biding to the $50 \mathrm{~S}$ ribosomal subunit $[61,56]$. The exact mode of acquisition of resistance to Capreomycin and Viomycin is not totally clear; however, cross-resistance between Capreomycin and Viomycin has been reported [59].

$[60,62]$, reported mutations in tlyA gene plays key role in Viomycin and Capreomycin resistance. Amikacin and Kanamycin alters the 16S rRNA configuration, thus inhibiting protein synthesis, amikacin and kanamycin resistance are associated with mutational alterations in the rrs gene [63-66] conformational changes in the aminoglycoside acetyltransferase gene (eis), also contribute to Kanamycin resistance [58].

\section{Conclusion}

Drug resistance in Mycobacterium tuberculosis is not a product of a single homogeneous genetic unit. Rather it is as a result of frequent mutation in various genes which encode for resistance to antibiotics. Also, the slow metabolism during a prolonged dormant stage greatly enhances it resistance to drug, the waxy impermeable cell wall with the presence of numerous efflux pump are essential for withstanding the potency of antibiotics. Having an adequate knowledge on the molecular mechanisms of drug resistance in M. tuberculosis may be helpful in exploring new targets for drug development.

\section{References}

1. Sotgiu G, Centis R, Dambrosio L, Migliori GB (2015) Tuberculosis treatment and drug regimens. Cold Spring Harb Perspect Med 5(5): a017822.

2. Hamusse SD, Teshome D, Hussen MS, Demissie M, Lindtjørn B (2016) Primary and secondary anti-tuberculosis drug resistance in Hitossa District of Arsi Zone, Oromia Regional State, Central Ethiopia. BMC public health 16: 593.

3. Li D, He W, Chen B, Lv P (2017) Primary multidrug-resistant tuberculosis versus drug-sensitive tuberculosis in non-HIV-infected patients: Comparisons of CT findings. PloS one 12(6): e0176354.

4. Böttger (2011) Drug resistance in mycobacterium tuberculosis:molecular mechanism and Laboratory susceptibility testing Donald PR, van Helden PD (eds) Antituberculosis Chemotherapy. Prog Respir Res 40: 128-144.

5. Maitra A, Munshi T, Healy J, Martin LT, Vollmer W, et al. (2019) Cell wall peptidoglycan in Mycobacterium tuberculosis: An Achilles' heel for the TB-causing pathogen. FEMS Microbiol Rev 43(5): 548-575.

6. Erik C Hett, Eric J Rubin (2015) Bacterial Growth and Cell Division: A Mycobacterial Perspective. Microbiol Mol Biol Rev 72(1): 126-156.

7. Danilchanka O, Pires D, Anes E, Niederweis (2015) The Mycobacterium tuberculosis Outer Membrane Channel Protein CpnT Confers
Susceptibility to Toxic Molecules. Antimicrob Agents Chemother 59(4): 2328-2336.

8. Chiaradia L, Lefebvre J, Marcoux J, Burlet Schiltz O, Etienne G, et al. (2017) Dissecting the mycobacterial cell envelope and defining the composition of the native mycomembrane. Scientific Reports 7(1): 12807.

9. Pandey AK, Sassetti C M (2008) Mycobacterial persistence requires the utilization of host cholesterol. Proc Natl Acad Sci USA 105(11): 43764380 .

10. Raffetseder J, Pienaar E, Blomgran R, Eklund D, Brodin VP, et al. (2014) Replication Rates of Mycobacterium tuberculosis in Human Macrophages Do Not Correlate with Mycobacterial Antibiotic Susceptibility. PLOS One 9(11): e112426

11. Goswam A, Chakraborty U, Bhattacharya B, Pal NK (2016) Association of generation time with anti-tubercular $\operatorname{drug}(\mathrm{s})$ resistance pattern of mycobacterium tuberculosis isolates among treatment failure pulmonary tuberculosis patients. Asian Journal of pharmaceutical and clinical Research 9(1): 258-261.

12. BaekSH, Li A H, Sassetti CM (2011) Metabolic Regulation of Mycobacterial Growth and Antibiotic Sensitivity. PLoS Biol 9(5): e1001065.

13. Daniel J, Deb C, Dubey VS, Sirakova TD, Abomoelak B (2010) Induction of a novel class of diacylglycerol acyltransferases and triacylglycerol accumulation in Mycobacterium tuberculosis as it goes into a dormancylike state in culture. J Bacteriol 186(15): 5017-5030.

14. Baker JJ, Abramovitch RB (2018) Genetic and metabolic regulation of Mycobacterium tuberculosis acid growth arrest. Sci Rep 8(1): 4168.

15. Warner D F (2014) Mycobacterium tuberculosis metabolism. Cold Spring Harb Perspect Med 5(4): a021121.

16. Nguyen L (2016) Antibiotic resistance mechanisms in M. tuberculosis: an update. Arch Toxicol 90(7): 1585-1604.

17. Knutson KL, Hmama Z, Herrera Velit P, Rochford R, Reiner NE (2014) Lipoarabinomannan of Mycobacterium tuberculosis promotes protein tyrosine dephosphorylation and inhibition of mitogen-activated protein kinase in human mononuclear phagocytes: role of the Src homology 2 containing tyrosine phosphatase 1 . Journal of Biological Chemistry 273(1): 645-652.

18. Kochi A, Vareldzis B, Styblo K (1993) Multi-Drug resistant tuberculosis and control. Resistant Microbiology 144: 104-110.

19. Mitchison DA (2015) Mechanism of drug action in short course chemotherapy. Bulletin mycobacteria by ethambutol. Lancet ii:1418.

20. Damtie D, Woldeyohannes D, Mathewos B (2014) Review on Molecular mechanism of first line antibiotic resistance in Mycobacterium tuberculosis. Mycobact Dis 4(6): 174.

21. De Vos M, Müller B, Borrell S, Black PA, van Helden PD, et al. (2013) Putative compensatory mutations in the rpoC gene of rifampin-resistant Mycobacterium tuberculosis are associated with ongoing transmission. Antimicrob Agents Chemother 57(2): 827-832.

22. Somoskovi A, Parsons LM, Salfinger M (2001) The molecular basis of resistance to isoniazid, rifampin, and pyrazinamide in Mycobacterium tuberculosis. Respir Res 2(3): 164-168.

23. Heep M, Rieger U, Beck D, Lehn N (2000) Mutations in the beginning of the rpoB gene can induce resistance to rifamycins in both Helicobacter pylori and Mycobacterium tuberculosis. Antimicrob Agents Chemother 44(4): 1075-1077.

24. Telenti A, Imboden P, Marchesi F, Lowrie D, Cole S, et al. (1993) Detection of rifampicin-resistance mutations in Mycobacterium tuberculosis. Lancet 341(8846): 647-650.

25. Kapur V, Li LL, Iordanescu S, Hamrick MR, Wanger A, et al. (1994) Characterization by automated DNA sequencing of mutations in the gene (rpoB) encoding the RNA polymerase beta subunit in rifampin- 
resistant Mycobacterium tuberculosis strains from New York City and Texas. J Clin Microbiol 32(4): 1095-1098.

26. Brandis G, Hughes D (2013) Genetic characterization of compensator evolution in strains carrying rpoB Ser531Leu, the rifampicin resistance mutation most frequently found in clinical isolates. J Antimicrob Chemother 68(11): 2493-2497.

27. Yang B, Koga H, Ohno H, Ogawa K, Fukuda M, et al. (1998) Relationship between antimycobacterial activities of rifampicin, rifabutin and KRM1648 and rpoB mutations of Mycobacterium tuberculosis J Antimicrob Chemother 42(5): 621-628.

28. Cavusoglu C, Karaca Derici Y, Bilgic A (2004) In-vitro activity of rifabutin against rifampicin-resistant Mycobacterium tuberculosis isolates with known rpoB mutations. Clin Microbiol Infect 10(7): 662-665.

29. Burman WJ, Jones BE (2001) Treatment of HIV-related tuberculosis in the era of effective antiretroviral therapy. Am J Respir Crit Care Med 164(1): 7-12

30. Tahir MK, Nayyer M, Sheed KA, Tanwir AM, Iqbal SM, et al. (2019) Pyrazinamide resistance and mutations in pncA among isolates of Mycobacterium tuberculosis from Khyber Pakhtunkhwa, Pakistan. BMC Infect Dis 19(1): 116.

31. Shi W, Zhang X, Jiang X, Yuan H, Lee JS, et al. (2011) Pyrazinamide inhibits trans-translation in Mycobacterium tuberculosis. Science 333(6049): 1630- 1632.

32. Njire M Tan Y, Mugweru J, Wang C, Guo J, Yew W, et al. (2016) Pyrazinamide resistance in Mycobacterium tuberculosis: Review and update. Adv Med Sci 61(1): 63-71.

33. Huy NQ, Lucie C, Hoa T, Hung NV, Lan N, et al. (2017) Molecular analysis of pyrazinamide resistance in Mycobacterium tuberculosis in Vietnam highlights the high rate of pyrazinamide resistance-associated mutations in clinical isolates. Emerg Microbes Infect 6(10): e86.

34. Zhang YJ, Li XJ, Mi KX (2016) Mechanisms of fluoroquinolone resistance in Mycobacterium tuberculosis. Yi Chuan 38(10): 918-927.

35. Johnsson K, Froland WA, Schultz PG (2007) Overexpression, purification and characterization of the catalaseperoxidase, katG from Mycobacterium tuberculosis. J Biol Chem 272(5): 2834-2840.

36. Bollela VR, Namburete EI, Feliciano CS, Macheque D, Harrison LH, et al. (2016) Detection of katG and inhA mutations to guide isoniazid and ethionamide use for drug-resistant tuberculosis. Int J Tuberc Lung Dis 20(8): 1099-1104.

37. Lempens P, Meehan CJ, Vandelannoote K, Fissette K, Rijk P, et al. (2018) Isoniazid resistance levels of Mycobacterium tuberculosis can largely be predicted by high-confidence resistance-conferring mutations. Scientific Reports 8: 3246.

38. Kandler JL, Mercante AD, Dalton TL, Ezewudo M, Cowan SL, et al. (2018) Validation of Novel Mycobacterium tuberculosis Isoniazid Resistance Mutations Not Detectable by Common Molecular Tests. Antimicrob Agents Chemother 62(10): e00974-e01018.

39. Hazbón MH, Brimacombe M, Bobadilla del Valle M, Cavatore M, Guerrero MI, et al. (2006) Population genetics study of isoniazid resistance mutations and evolution of multidrug-resistant Mycobacterium tuberculosis. Antimicrob Agents Chemother 50(8): 2640-2649.

40. Seifert M, Catanzaro D, Catanzaro A, Rodwell TC (2015) Genetic mutations associated with isoniazid resistance in Mycobacterium tuberculosis: a systematic review. PloS one 10(3): e0119628.

41. Chesov D, Ciobanu N, Lange C, Schön T, Heyckendorf J, et al. (2017) Lack of evidence of isoniazid efficacy for the treatment of MDR/XDR-TB in the presence of the katG 315T mutation. Eur Respir J 50(4): 1701752.

42. Parsons LM, Salfinger M, Clobridge A, Dormandy J, Mirabello L, et al. (2005) Phenotypic and Molecular Characterization of Mycobacterium tuberculosis Isolates Resistant to both Isoniazid and Ethambutol. Antimicrob Agents Chemother 49(6): 2218-2225.
43. Ando H, Miyoshi Akiyama T, Watanabe S, Kirikae T (2014) A silent mutation in mabA confers isoniazid resistance on Mycobacterium tuberculosis. Mol Microbiol 91(3): 538-547.

44. Poso H, Paulin L, Brander E (1983) Specific inhibition of spermidine synthase from mycobacteria by ethambutol. Lancet 2(8364): 1418.

45. Safi H, Lingaraju S, Amin A (2013) Evolution of high-level ethambutol-resistant tuberculosis through interacting mutations in decaprenylphosphoryl- $\beta$-D-arabinose biosynthetic and utilization pathway genes. Nat Genet 45(10): 1190-1197.

46. Hazbón MH, Bobadilla del Valle M, Guerrero MI, Varma Basil M, Filliol I, et al. (2005) Role of embB codon 306 mutations in Mycobacterium tuberculosis revisited: A novel association with broad drug resistance and IS 6110 clustering rather than ethambutol resistance. Antimicrob Agents Chemother 49(9): 3794-3802.

47. Gale E F, Cundliffe E Reynolds PE, Richmond MH, Waring M J (1981) The molecular basis of antibiotic action. 62(9): 1577-1578.

48. Plinke C, Cox HS, Zarkua N, Karimovich HA, Braker A, et al. (2010) embCAB sequence variation among ethambutol-resistant Mycobacterium tuberculosis isolates without embB306 mutation. J Antimicrob Chemother 65(7):1359-1367.

49. Ahmad S, Jaber AA, Mokaddas E (2007) Frequency of embB codon 306 mutations in Ethambutol-susceptible and -resistant clinical Mycobacterium tuberculosis isolates in Kuwait. Tuberculosis 87(2): 123-129.

50. Al Mutairi NM, Ahmad S, Mokaddas E (2018) Molecular Screening Versus Phenotypic Susceptibility Testing of Multidrug-Resistant Mycobacterium tuberculosis Isolates for Streptomycin and Ethambutol. Microb Drug Resist 24(7): 923-931.

51. Tulyaprawat O, Chaiprasert A, Chongtrakool P, Suwannakarn K Ngamskulrungroj P (2019) Association of ubiA mutations and highlevel of ethambutol resistance among Mycobacterium tuberculosis Thai clinical isolates. Tuberculosis 114: 42-46.

52. Lingaraju S, Rigouts L, Gupta A, Lee J, Umubyeyi AN, et al. (2016) Geographic differences in the contribution of ubiA mutations to highlevel ethambutol resistance in Mycobacterium tuberculosis. Antimicrob Agents Chemother 60(7): 4101-4105.

53. Palomino JC, Martin A (2013) Tuberculosis clinical trial update and the current anti- tuberculosis drug portfolio. Curr Med Chem 20(30): 3785 3796.

54. Bernard C, Veriris N, Brossier F, Sougakoff jarlier V, Robert J, et al. (2018) Molecular Diagnosis of Fluoroquinolone Resistance in Mycobacterium tuberculosis. Antimicrob Agents Chemother 59(3): 1519-1524.

55. Che Y, Song Q, Yang T, Ping G, Yu G (2107) Fluoroquinolone resistance in multidrug-resistant Mycobacterium tuberculosis independent of fluoroquinolone use. Eur Respir J 50(6): 1701633.

56. Carette X, Blondiaux N, Willery E, Hoos S, Lecat Guillet N, et al. (2012) Structural activation of the transcriptional repressor EthR from Mycobacterium tuberculosis by single amino acid change mimicking natural and synthetic ligands. Nucleic Acids Res 40(7): 3018-3030.

57. Vilchèze C, Av Gay Y, Attarian R, Liu Z, Hazbón MH, et al. (2008) Mycothiol biosynthesis is essential for ethionamide susceptibility in Mycobacterium tuberculosis. Mol Microbiol 69(5): 1316-1329.

58. Brossier F, Veziris N, Truffot Pernot C, Jarlier V, Sougakoff W (2011) Molecular investigation of resistance to the antituberculous drug ethionamide in multidrug-resistant clinical isolates of Mycobacterium tuberculosis. Antimicrob Agents Chemother 55(1): 355-560.

59. De Barber AE, Mdluli K, Bosman M, Bekker LG, Barry CE (2000) Ethionamide activation and sensitivity in multidrug-resistant Mycobacterium tuberculosis. Proc Natl Acad Sci USA 97(17): 9677-9782.

60. Johansen SK, Maus CE, Plikaytis BB, Douthwaite S (2006) Capreomycin binds across the ribosomal subunit interface using tlyA -encoded 2'-0-methylations in 16S and 23S rRNAs. Molecular Cell 23: 173-182. 
61. Stanley RE, Blaha G, Grodzicki, RL, Strickler MD, Steitz TA (2010) The structures of the anti-tuberculosis antibiotics viomycin and capreomycin bound to the 70S ribosome. Nat Struct Mol Biol 17(3): 289-293.

62. Georghiou SB, Magana M, Garfein RS, Catanzaro DG, Catanzaro A, et al (2012) Evaluation of genetic mutations associated with Mycobacterium tuberculosis resistance to amikacin, kanamycin and capreomycin: A systematic review. PLoS One 7(3): e33275.

63. Zaunbrecher MA, Sikes RD, Metchock B, Shinnick TM, Posey JE (2009) Overexpression of the chromosomally encoded aminoglycoside acetyltransferase eis confers kanamycin resistance in Mycobacterium tuberculosis. Proc Natl Acad Sci USA 106(47): 20004-20009.
64. Campbell PJ, Morlock GP, Sikes RD, Dalton TL, Metchock B, et al. (2011) Molecular detection of mutations associated with first- and second-line drug resistance compared with conventional drug susceptibility testing of Mycobacterium tuberculosis. Antimicrob Agents Chemother 55(5): 2032-2041.

65. Caws M, Duy PM, Tho DQ, Lan NT, Hoa DV, et al. (2006) Mutations prevalent among rifampin and isoniazid-resistant Mycobacterium tuberculosis isolates from a hospital in Vietnam. J Clin Microbiol 44(7): 2333-2337.

66. Christopher A Kerantzas, William R Jacobs (2017) Origins of Combination Therapy for Tuberculosis: Lessons for Future Antimicrobial Development and Application. mBio 8(2): e01586-e01616. 